\title{
PERFormance OF A High COUnT RATE Silicon DRIFT X-RAY DETECTOR ON THE ANL 300 KV ADVANCED ANALYTICAL ELECTRON MiCROSCOPE.
}

\author{
N. J. ZAluzec ${ }^{1}$, J. S. IWAnCZYK ${ }^{2}$, B. E. PATT ${ }^{2}$, S. BARKAN ${ }^{2}$, L. Feng ${ }^{2}$ \\ ${ }^{1-}$ Argonne National Laboratory, Electron Microscopy Center, Materials Science Div., Argonne, Il, USA \\ ${ }^{2-}$ Photon Imaging Inc., 19355 Business Center Drive, Suite \# 8, Northridge, Ca. USA
}

Silicon Drift Detectors (SDD), were originally developed for use in high energy physics experiments, however, in recent years, they have been transformed to extend their operational mode as X-ray Energy Dispersive Spectrometers (XEDS) systems [1-3]. Three of the most important reasons for this development are: 1.) their relatively large size $\left(\sim 1 \mathrm{~cm}^{2}\right)$ yields large solid angles, 2 .) an ability to operate at near room temperature removing the need for $\mathrm{LN}_{2}$, and 3.) their inherently low capacitance which enables high counting rates. This new breed of detectors has, to-date, been incorporated into X-ray Diffractometers, X-ray Fluorescence Spectrometers, and Scanning Electron Microscopes [4-5], however, no work has yet been reported on their use in the AEM/TEM/STEM instruments. The purpose of this work is to evaluate the performance of the SDD on an intermediate voltage AEM, particularly with respect to potential applications in the US DoE TEAM project [6].

The experimental work reported herein has been carried out using the ANL AAEM HB603Z CFEG instrument [7]. The detector employed was a, customized, windowless $50 \mathrm{~mm}^{2}$, hexagonal shaped Vortex ${ }^{\mathrm{TM}}$ detector from Photon Imaging Inc, (figure 1). The detector was mounted on the AAEM in place of a the standard $\mathrm{Si}(\mathrm{Li})$ detector at an elevation angle of 20 degrees to the incident beam surface (Fig. 2). In order to determine the characteristics of this detector under a wide range of conditions, both sample thickness and beam current variation were used to stress the SDD system. Variation of the incident beam current was accomplished by a combination of changing the FEG extraction voltage and/or the size of the probe forming apertures. Thickness was varied by simply translating the specimen from thin to bulk areas. All data was generated at $300 \mathrm{kV}$, while spectra were record and stored using PI-Spec DAQ program on a Win2K PC, then transferred to a Macintosh OS X system for detailed analysis.

Figure 3 presents the experimentally measured energy resolution as a function of processing time constant (Tc). Interpolating to $5.9 \mathrm{keV}$, the resolution of this detector ranges from 146 to $300 \mathrm{eV}$ and, as in all such devices, we see that the resolution improves with processing; decreasing as Tc increases from $250 \mathrm{~ns}$ to 64 Hs. This compares favorably with the SDD off-column resolution of $143 \mathrm{eV} \mathrm{FWHM.} \mathrm{At} \mathrm{the} \mathrm{present}$ time, we believe that the existence of the high energy electrons flux in the AAEM contribute substantially to the electronic noise reducing the resolution. This was confirmed by operating the AAEM system with the Objective Lens off, eliminating the effective magnetic trap and observing the corresponding deterioration in performance. In figure 4, we plot the experimental resolution performance as a function of input count rate. Here, we find no significant change in resolution for input count rates less than 150 kcps, while above $200 \mathrm{kcps}$, we begin to see a resolution degradation for all energies investigated. Figure 5, and 6 present representative spectral profiles taken from a semiconductor specimen mounted on a Molybdenum support ring. In order to excite both high and low lines, the sample was intentionally translated so that the incident probe excited both the specimen and it's Mo support ring. Figure 5, shows the low energy regime (0-3 keV) performance, for 3 time constants of 16, 2, $0.5 \mu \mathrm{sec}$, while figure 6 does the similar for high energies (16-21 keV). For time constants $\geq 2 \mu \mathrm{sec}$ the light element detection capabilities readily permit measurement of Oxygen $\mathrm{K}$ at $532 \mathrm{eV}$, but as the time constant is decreased to accommodate very high count rates, the increased electronic noise restricts low energy peak detection. The high energy performance of the detector is satisfactory at all times. Additional work is in progress to assess the functionality of this detector in a number of modes and to measure its radiation damage resistance.

References:

[1] E. Gatti and P. Rehak, Nucl. Instr. Meth., Phys. Res. 225, 608 (1984).

[2] G. Bertuccio, etal, Nucl. Instr. Meth., Phys. Res. A312, 613 (1992).

[3] J.S. Iwanczyk, etal, Nucl. Instr. \& Meth. in Phys. Res. A380 (1996) 288-294. 
[4] J.S. Iwanczyk,etal , Proc. of Microscopy \& Microanalaysis 2001, Vol 7, sup. 2, p1052 Long Beach

[5] P. Smith, etal , Proc. of Microscopy \& Microanalaysis 2001, Vol 7, sup. 2, p 1054 Long Beach,

[6] B. Kabius etal, Proc. of Microscopy \& Microanalaysis 2002, Vol 8, sup. 2, p 418 Quebec City,

[7] N.J. Zaluzec, Microbeam Analysis, Proc. of $26^{\text {th }}$ Annual Conf. San Francisco Press. p137, (1991)

[8] This work was supported in part by the U.S. DoE under BES-MS W-31-109-Eng-38 at ANL and a DoE SBIR Grant \# DE-FG03-02ER83548 at Photon Imaging Inc.

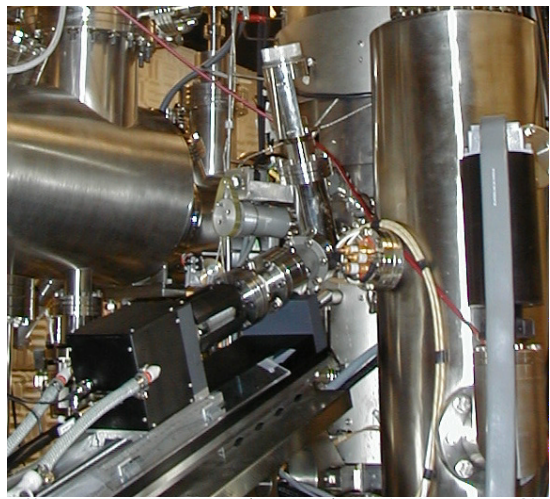

Fig.1. SDD mounted on the AAEM Column

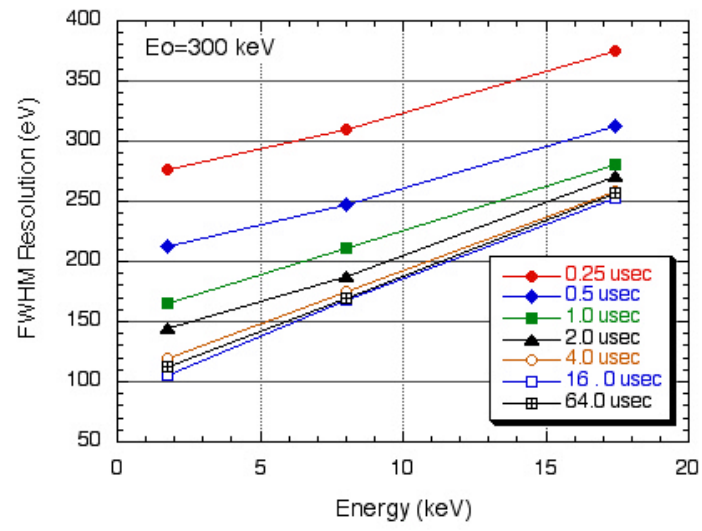

Fig. 3. Resolution of the SDD/AAEM system as a function of processor Tc

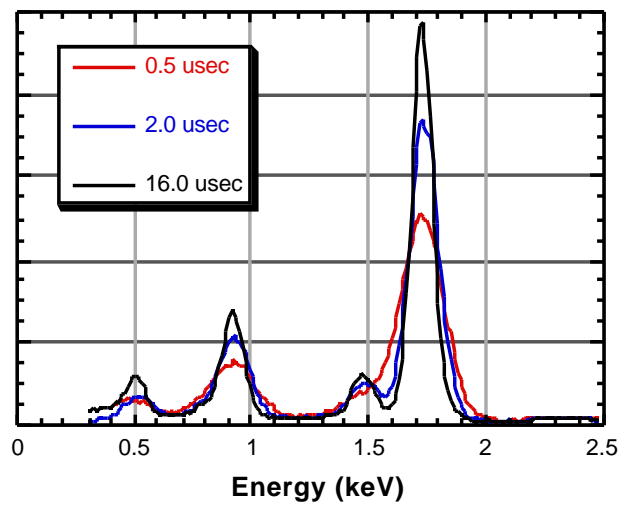

Fig. 5. Low energy performance (SiK, AlK, $\mathrm{CuL}, \mathrm{OK}$ ) of the SDD/AAEM system as a function of processor time constant.

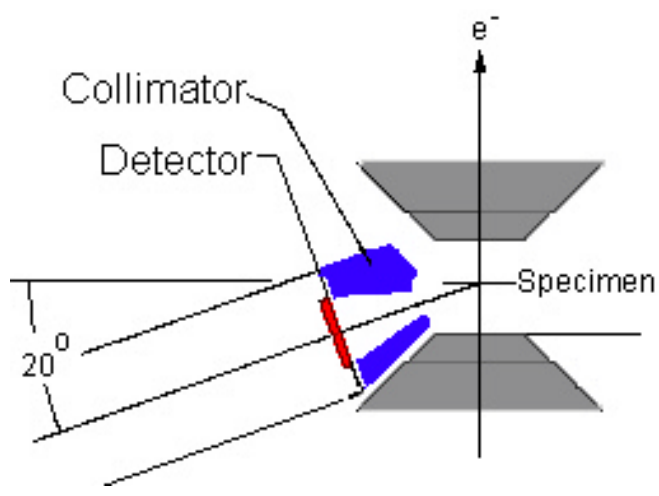

Fig. 2. Detector/Specimen Geometry

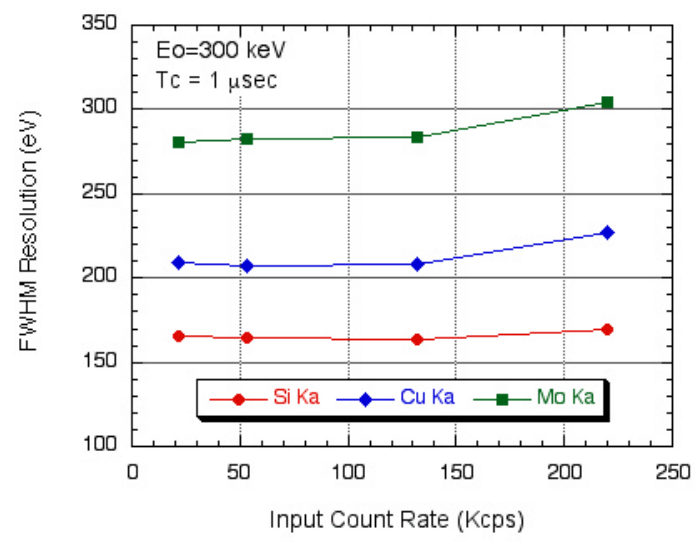

Fig. 4. Resolution of the SDD/AAEM system $(\mathrm{Tc}=1 \mu \mathrm{sec})$ as a function of input count rate.

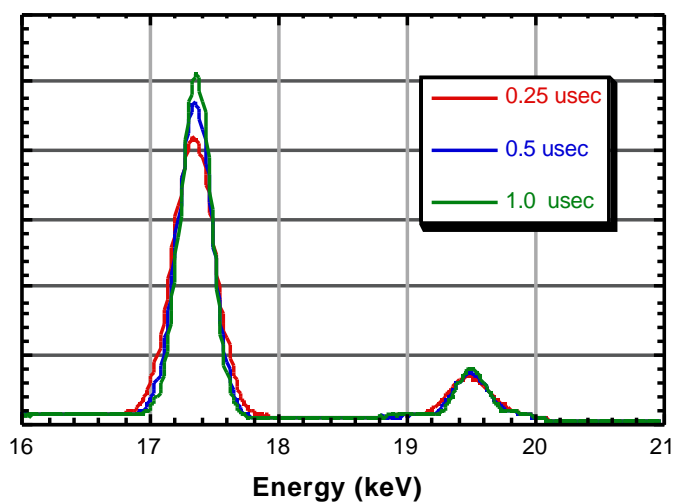

Fig. 6. High energy $(\mathrm{MoK} \alpha / \mathrm{K} \beta)$ performance of the SDD/AAEM system as a function of processor time constant. 